\title{
15. CARBON CARBONATE RESULTS, LEG 3
}

\author{
A. C. Pimm, Deep Sea Drilling Project, East Coast Repository \\ Lamont-Doherty Geological Observatory, Palisades, N. Y.
}

\section{INTRODUCTION}

The methods used in determining the total carbon, organic carbon, and calcium carbonate percentages have been described in the Leg 1 cruise report.

Nine of the 10 holes drilled on Leg 3 were aligned approximately along latitude $29^{\circ}$ South, thus giving a section across the Mid-Atlantic Ridge and the Rio Grande Rise. These nine holes all penetrated mainly foraminiferal-nannofossil chalk oozes with a high calcium carbonate content. The other hole was drilled on the Sierre Leone Rise and the sediments here contained less carbonate.

The complete carbon carbonate results are given as Table 1 and average values for each hole as Table 2.

\section{CALCIUM CARBONATE}

\section{Results}

The results show that although most of the South Atlantic sediments have high carbonate values, there are important variations as follows:

(1) Sediments with the highest carbonate content occur closest to the ridge axis, the average value in Hole 16 being 89 per cent.

(2) Sediments to the east of the Mid-Atlantic Ridge (Holes 17 and 18), have higher carbonate values than those sediments in comparative positions on the west side of the ridge (Holes 14 and 15), 82 per cent carbonate as against 71 per cent.

(3) There is little variation in carbonate content down the flanks of the ridge, for example, Holes 14 and 15 have carbonate values only 2 to 4 per cent different from Holes 19 and 29.

(4) Sediments from the Rio Grande Rise (Holes 21 and 22) have carbonate contents of 78 to 79 per cent, i.e. some 4 per cent higher than sediments from the west flank of the ridge, but still 10 per cent lower than those on the crest of the ridge.

\section{Discussion}

Comparing these results with the carbonate results from Leg 2 in the North Atlantic, it can be seen that there is quite a close correlation. Hole 10 on Leg 2 had an average carbonate content of 69 per cent compared with 71 per cent for Hole 14 on Leg 3, both holes being about the same distance down the west flank of the Mid-Atlantic Ridge. Again, sediments close to the crest of the ridge had the highest carbonate values in both the North and South Atlantic; though in the former, values are about 6 per cent lower (see Table 2). No holes were drilled on the east flank of the ridge in the North Atlantic, so it is not possible to make any comparisons here.

From an examination of the results in Table 2, there does not appear to be any direct correlation between carbonate content and present oceanic depth (above the calcium carbonate compensation depth) or carbonate content in relation to age of the sediments.

The highest carbonate values on the crestal region of the ridge would appear to be related to the high rate of accumulation of chalk oozes on the crest, as compared with the flanks of the ridge. Sediments accumulate about three times faster on the ridge crest than on the flanks (see elsewhere in this cruise report). Therefore, it appears that the slower rate of accumulation of sediment on the ridge flanks allows for a greater dilution of the carbonate content with other constituents. There also may be some partial solution of carbonate, particularly in the upper part of the sedimentary sections on the west side of the ridge, as most of this area is presently below the carbonate compensation depth.

It can be seen from the results that average calcium carbonate values (for sediments mainly ranging in age from Oligocene to Pliocene) are about 10 to 12 per cent higher on the east side of the Mid-Atlantic Ridge than on the west. It is known that at present times the calcium carbonate compensation depth is much deeper on the east side of the ridge-5000+ meters $(16,400+$ feet) as against 4000 meters $(13,120$ feet) for the west side. This is due to the effects of the Antarctic bottom water that sweeps the floor of the west flank of the Mid-Atlantic Ridge, but which is prevented from reaching the east flank by the ridge crest and the Walvis Ridge. The fast-flowing, cold Antarctic water is strongly undersaturated with respect to calcium carbonate. East of the ridge there is just a sluggish circulation of warmer Atlantic intermediate water, which is not undersaturated with respect to calcite until depths in excess of 5000 meters $(16,400$ feet $)$. 
TABLE 1

Leg 3, Carbon Carbonate Results

\begin{tabular}{|c|c|c|c|c|c|c|}
\hline Hole & Core & Section & $\begin{array}{l}\text { Sampled } \\
\text { at }(\mathrm{cm})\end{array}$ & $\begin{array}{c}\text { Total Carbon } \\
\text { Per Cent }\end{array}$ & $\begin{array}{l}\text { Organic Carbon } \\
\text { Per Cent }\end{array}$ & $\begin{array}{c}\text { Calcium Carbonate } \\
\text { Per Cent }\end{array}$ \\
\hline 13 & 1 & 1 & 50 & 6.3 & 0.3 & 50.3 \\
\hline 13 & 1 & 2 & $46.5-47.5$ & 7.8 & 0.2 & 63.6 \\
\hline 13 & 1 & 3 & $44-46$ & 6.4 & 0.3 & 51.1 \\
\hline 13 & 1 & 4 & $46-48$ & 6.7 & 0.3 & 53.1 \\
\hline 13 & 1 & 5 & $50-51$ & 6.4 & 0.4 & 49.6 \\
\hline 13 & 1 & 6 & $48-50$ & 10.0 & 0.5 & 78.8 \\
\hline 13 & 2 & 1 & $?$ & 7.0 & 0.3 & 55.6 \\
\hline 13 & 2 & 2 & $38-40$ & 3.0 & 0.3 & 22.5 \\
\hline 13 & 2 & 3 & $79-81$ & 5.6 & 0.2 & 45.3 \\
\hline 13 & 2 & 4 & $51-53$ & 0.5 & 0.2 & 2.2 \\
\hline 13 & 2 & 6 & $88-90$ & 0.4 & 0.4 & 0.0 \\
\hline 13 & 3 & 1 & $72-73$ & 0.3 & 0.2 & 1.0 \\
\hline 13 & 3 & 2 & $42-44$ & 0.5 & 0.2 & 2.7 \\
\hline 13 & 3 & 3 & $50-52$ & 0.4 & 0.3 & 1.1 \\
\hline 13 & 3 & 5 & $45-47$ & 0.4 & 0.3 & 0.5 \\
\hline 13 & 3 & 6 & $63-64$ & 0.4 & 0.3 & 0.7 \\
\hline $13 \mathrm{~A}$ & 1 & 1 & $115-116$ & 4.9 & 0.3 & 38.7 \\
\hline $13 \mathrm{~A}$ & 2 & 1 & $99-100$ & 4.1 & 0.3 & 32.0 \\
\hline $13 \mathrm{~A}$ & 3 & 1 & 100 & 2.2 & 0.2 & 16.5 \\
\hline $13 \mathrm{~A}$ & 3 & 3 & 47 & 3.2 & 0.4 & 23.0 \\
\hline $13 \mathrm{~A}$ & 3 & 4 & 57 & 3.1 & 0.3 & 23.0 \\
\hline $13 \mathrm{~A}$ & 5 & 1 & 52 & 1.0 & 0.3 & 5.7 \\
\hline 14 & $1 \mathrm{~A}$ & 1 & $?$ & 6.6 & 0.2 & 53.0 \\
\hline 14 & $1 \mathrm{~A}$ & 2 & $41-42$ & 8.6 & 0.1 & 71.1 \\
\hline 14 & $1 \mathrm{~A}$ & 3 & 45 & 7.9 & 0.1 & 64.8 \\
\hline 14 & $1 \mathrm{~A}$ & 4 & $48-50$ & 3.7 & 0.1 & 29.6 \\
\hline 14 & $1 \mathrm{~A}$ & 5 & $47-49$ & 7.7 & 0.1 & 63.1 \\
\hline 14 & $1 \mathrm{~A}$ & 6 & $38-40$ & 7.8 & 0.1 & 64.1 \\
\hline 14 & 2 & 1 & $54-55$ & 9.1 & 0.1 & 74.8 \\
\hline 14 & 2 & 2 & $49-50$ & 9.1 & 0.1 & 75.1 \\
\hline 14 & 2 & 3 & $46-48$ & 9.0 & 0.1 & 74.3 \\
\hline 14 & 2 & 4 & $51-53$ & 9.9 & 0.1 & 81.6 \\
\hline 14 & 2 & 5 & $49-50$ & 8.9 & 0.1 & 73.5 \\
\hline 14 & 3 & 2 & $47-48$ & 9.3 & 0.0 & 77.5 \\
\hline 14 & 3 & 3 & $51-52$ & 9.2 & 0.1 & 76.1 \\
\hline 14 & 3 & 4 & $46-47$ & 9.7 & 0.2 & 79.1 \\
\hline 14 & 3 & 5 & $47-48$ & 9.0 & 0.1 & 74.3 \\
\hline 14 & 3 & 6 & $48-50$ & 9.4 & 0.1 & 77.2 \\
\hline
\end{tabular}


TABLE 1 - Continued

\begin{tabular}{|c|c|c|c|c|c|c|}
\hline Hole & Core & Section & $\begin{array}{l}\text { Sampled } \\
\text { at }(\mathrm{cm})\end{array}$ & $\begin{array}{c}\text { Total Carbon } \\
\text { Per Cent }\end{array}$ & $\begin{array}{l}\text { Organic Carbon } \\
\text { Per Cent }\end{array}$ & $\begin{array}{c}\text { Calcium Carbonate } \\
\text { Per Cent }\end{array}$ \\
\hline 14 & 5 & 1 & $58-59$ & 9.2 & 0.3 & 74.1 \\
\hline 14 & 5 & 2 & $47-48$ & 9.9 & 0.0 & 82.8 \\
\hline 14 & 5 & 4 & $47-48$ & 9.7 & 0.0 & 80.6 \\
\hline 14 & 5 & 5 & $46-47$ & 9.3 & 0.0 & 77.3 \\
\hline 14 & 5 & 6 & $46-47$ & 9.1 & 0.1 & 75.1 \\
\hline 15 & 1 & 1 & $45-46$ & 9.8 & 0.1 & 80.8 \\
\hline 15 & 1 & 2 & $46-47$ & 9.2 & 0.1 & 75.7 \\
\hline 15 & 1 & 5 & $47-48$ & 9.4 & 0.1 & 77.6 \\
\hline 15 & 2 & 1 & $50-51$ & 4.8 & 0.2 & 38.4 \\
\hline 15 & 2 & 2 & $47-48$ & 9.6 & 0.0 & 79.7 \\
\hline 15 & 2 & 3 & $50-51$ & 9.8 & 0.0 & 81.7 \\
\hline 15 & 2 & 4 & $50-51$ & 9.7 & 0.0 & 80.7 \\
\hline 15 & 2 & 5 & $50-51$ & 10.6 & 0.0 & 88.3 \\
\hline 15 & 2 & 6 & $50-51$ & 9.6 & 0.0 & 79.6 \\
\hline 15 & 3 & 1 & $50-51$ & 10.3 & 0.0 & 85.8 \\
\hline 15 & 3 & 3 & $50-51$ & 11.1 & 0.0 & 92.5 \\
\hline 15 & 3 & 4 & $49-50$ & 11.0 & 0.0 & 91.6 \\
\hline 15 & 3 & 5 & $47-48$ & 11.2 & 0.0 & 93.3 \\
\hline 15 & 3 & 6 & $50-51$ & 10.7 & 0.0 & 89.1 \\
\hline 15 & 4 & 1 & $42-43$ & 11.0 & 0.0 & 91.6 \\
\hline 15 & 4 & 2 & $53-54$ & 10.9 & 0.0 & 90.8 \\
\hline 15 & 4 & 3 & $50-51$ & 11.0 & 0.0 & 91.6 \\
\hline 15 & 4 & 4 & $50-51$ & 11.0 & 0.0 & 91.6 \\
\hline 15 & 4 & 5 & $47-48$ & 11.0 & 0.0 & 91.6 \\
\hline 15 & 4 & 6 & $50-51$ & 10.5 & 0.0 & 87.5 \\
\hline 15 & 5 & 1 & $50-51$ & 9.0 & 0.0 & 75.0 \\
\hline 15 & 5 & 2 & $47-48$ & 9.7 & 0.0 & 80.8 \\
\hline 15 & 5 & 3 & $50-51$ & 9.1 & 0.1 & 75.8 \\
\hline 15 & 5 & 4 & $50-51$ & 10.1 & 0.0 & 84.1 \\
\hline 15 & 5 & 5 & $45-46$ & 9.7 & 0.1 & 80.8 \\
\hline 15 & 5 & 6 & $50-51$ & 10.1 & 0.0 & 84.1 \\
\hline 15 & 6 & 2 & $61-62$ & 8.4 & 0.1 & 70.0 \\
\hline 15 & 6 & 3 & $50-51$ & 4.6 & 0.1 & 38.3 \\
\hline 15 & 6 & 4 & $50-51$ & 3.9 & 0.1 & 32.5 \\
\hline 15 & 6 & 5 & $51-52$ & 9.3 & 0.0 & 77.5 \\
\hline 15 & 6 & 6 & $50-51$ & 9.6 & 0.0 & 80.0 \\
\hline 15 & 7 & 1 & $50-51$ & 9.6 & 0.0 & 80.0 \\
\hline 15 & 7 & 2 & $45-46$ & 9.8 & 0.0 & 81.6 \\
\hline 15 & 7 & 3 & $50-51$ & 9.5 & 0.0 & 79.1 \\
\hline
\end{tabular}


TABLE 1 - Continued

\begin{tabular}{|c|c|c|c|c|c|c|}
\hline Hole & Core & Section & $\begin{array}{l}\text { Sampled } \\
\text { at (cm) }\end{array}$ & $\begin{array}{c}\text { Total Carbon } \\
\text { Per Cent }\end{array}$ & $\begin{array}{l}\text { Organic Carbon } \\
\text { Per Cent }\end{array}$ & $\begin{array}{c}\text { Calcium Carbonate } \\
\text { Per Cent }\end{array}$ \\
\hline 15 & 7 & 4 & $50-51$ & 9.7 & 0.0 & 80.8 \\
\hline 15 & 7 & 5 & $50-51$ & 9.1 & 0.0 & 75.8 \\
\hline 15 & 7 & 6 & $50-51$ & 10.1 & 0.0 & 84.1 \\
\hline 15 & 8 & 2 & $45-46$ & 10.1 & 0.0 & 84.1 \\
\hline 15 & 8 & 3 & $50-51$ & 10.1 & 0.0 & 84.1 \\
\hline 15 & 8 & 4 & $50-51$ & 9.8 & 0.0 & 81.6 \\
\hline 15 & 8 & 5 & $42-43$ & 10.5 & 0.0 & 87.5 \\
\hline 15 & 8 & 6 & $50-51$ & 9.8 & 0.0 & 81.6 \\
\hline 15 & 9 & 2 & $47-48$ & 10.1 & 0.0 & 84.1 \\
\hline 15 & 9 & 3 & $50-51$ & 10.5 & 0.0 & 87.5 \\
\hline 15 & 9 & 4 & $50-51$ & 9.0 & 0.0 & 75.0 \\
\hline 15 & 9 & 5 & $52-53$ & 9.8 & 0.0 & 81.6 \\
\hline 15 & 9 & 6 & $50-51$ & 11.0 & 0.0 & 91.6 \\
\hline 16 & 1 & 1 & $50-51$ & 10.5 & 0.1 & 87.6 \\
\hline 16 & 1 & 2 & $56-57$ & 10.0 & 0.0 & 83.3 \\
\hline 16 & 1 & 4 & $0-3$ & 10.7 & 0.0 & 89.1 \\
\hline 16 & 2 & 5 & 150 & 10.5 & 0.0 & 87.5 \\
\hline 16 & 2 & 6 & $45-46$ & 10.6 & 0.0 & 88.3 \\
\hline 16 & 3 & 1 & 150 & 11.3 & 0.0 & 94.1 \\
\hline 16 & 3 & 2 & 150 & 11.6 & 0.0 & 96.6 \\
\hline 16 & 3 & 3 & 150 & 10.9 & 0.0 & 90.8 \\
\hline 16 & 3 & 4 & 150 & 11.2 & 0.0 & 93.3 \\
\hline 16 & 3 & 5 & 150 & 11.5 & 0.0 & 95.8 \\
\hline 16 & 3 & 6 & 150 & 11.0 & 0.0 & 91.6 \\
\hline 16 & 4 & 1 & $50-51$ & 10.8 & 0.0 & 90.0 \\
\hline 16 & 4 & 2 & $5-6$ & 11.2 & 0.0 & 93.3 \\
\hline 16 & 4 & 3 & $5-6$ & 10.7 & 0.0 & 89.1 \\
\hline 16 & 4 & 4 & $5-6$ & 11.4 & 0.0 & 95.0 \\
\hline 16 & 4 & 5 & $5-6$ & 11.4 & 0.0 & 95.0 \\
\hline 16 & 4 & 6 & 150 & 11.2 & 0.0 & 93.3 \\
\hline 16 & 5 & 1 & 150 & 10.9 & 0.0 & 90.8 \\
\hline 16 & 5 & 2 & 150 & 11.2 & 0.0 & 93.3 \\
\hline 16 & 5 & 3 & 150 & 9.4 & 0.2 & 76.6 \\
\hline 16 & 5 & 4 & 150 & 11.2 & 0.0 & 93.1 \\
\hline 16 & 5 & 5 & 150 & 10.0 & 0.0 & 90.5 \\
\hline 16 & 5 & 6 & 150 & 11.0 & 0.2 & 90.0 \\
\hline 16 & 6 & 1 & 150 & 11.1 & 0.0 & 92.5 \\
\hline 16 & 6 & 2 & 150 & 11.1 & 0.2 & 90.9 \\
\hline 16 & 6 & 3 & 150 & 11.1 & 0.1 & 91.6 \\
\hline
\end{tabular}


TABLE 1 - Continued

\begin{tabular}{|c|c|c|c|c|c|c|}
\hline Hole & Core & Section & $\begin{array}{l}\text { Sampled } \\
\text { at }(\mathrm{cm})\end{array}$ & $\begin{array}{c}\text { Total Carbon } \\
\text { Per Cent }\end{array}$ & $\begin{array}{l}\text { Organic Carbon } \\
\text { Per Cent }\end{array}$ & $\begin{array}{c}\text { Calcium Carbonate } \\
\text { Per Cent }\end{array}$ \\
\hline 16 & 6 & 4 & 150 & 10.5 & 0.0 & 87.6 \\
\hline 16 & 6 & 5 & 150 & 11.1 & 0.1 & 91.6 \\
\hline 16 & 6 & 6 & 150 & 11.0 & 0.2 & 90.0 \\
\hline 16 & 7 & 1 & 150 & 10.8 & 0.0 & 89.8 \\
\hline 16 & 7 & 2 & 150 & 10.6 & 0.1 & 87.5 \\
\hline 16 & 7 & 3 & 150 & 11.0 & 0.0 & 91.9 \\
\hline 16 & 7 & 4 & 150 & 11.3 & 0.1 & 92.9 \\
\hline 16 & 7 & 5 & 150 & 11.2 & 0.2 & 91.7 \\
\hline 16 & 7 & 6 & 150 & 10.8 & 0.0 & 89.6 \\
\hline 16 & 8 & 1 & 150 & 10.8 & 0.1 & 89.5 \\
\hline 16 & 8 & 2 & 150 & 10.6 & 0.1 & 87.3 \\
\hline 16 & 8 & 3 & 150 & 10.9 & 0.0 & 91.0 \\
\hline 16 & 8 & 4 & 150 & 10.9 & 0.0 & 90.6 \\
\hline 16 & 8 & 5 & 150 & 10.7 & 0.1 & 88.3 \\
\hline 16 & 8 & 6 & 150 & 11.6 & 0.1 & 95.4 \\
\hline 16 & 9 & 1 & 150 & 10.7 & 0.1 & 88.6 \\
\hline 16 & 9 & 2 & 150 & 10.5 & 0.0 & 87.8 \\
\hline 16 & 9 & 3 & 150 & 11.4 & 0.2 & 93.3 \\
\hline 16 & 9 & 4 & 150 & 10.8 & 0.2 & 88.1 \\
\hline 16 & 9 & 6 & 150 & 10.8 & 0.2 & 87.9 \\
\hline 16 & 10 & 1 & 150 & 11.4 & 0.1 & 94.2 \\
\hline 16 & 10 & 2 & 150 & 10.3 & 0.2 & 84.3 \\
\hline 16 & 10 & 3 & 150 & 11.6 & 0.1 & 95.4 \\
\hline 16 & 10 & 4 & 150 & 10.3 & 0.0 & 85.8 \\
\hline 16 & 10 & 5 & 150 & 11.2 & 0.2 & 91.7 \\
\hline 16 & 10 & 6 & 150 & 10.7 & 0.1 & 88.5 \\
\hline 16 & 11 & 1 & 150 & 11.4 & 0.1 & 94.2 \\
\hline 16 & 11 & 2 & 150 & 10.6 & 0.2 & 86.9 \\
\hline 16 & 11 & 3 & 150 & 11.5 & 0.2 & 93.9 \\
\hline 16 & 11 & 4 & 150 & 10.6 & 0.1 & 87.0 \\
\hline 16 & 11 & 5 & 150 & 10.9 & 0.0 & 90.7 \\
\hline 16 & 11 & 6 & 150 & 10.8 & 0.2 & 88.5 \\
\hline 17 & 1 & 1 & $25-26$ & 10.3 & 0.2 & 83.8 \\
\hline 17 & 1 & 2 & $44-45$ & 10.9 & 0.2 & 89.0 \\
\hline 17 & 1 & 3 & $2-6$ & 10.5 & 0.2 & 85.7 \\
\hline 17 & 1 & 4 & $5-6$ & 10.8 & 0.2 & 88.8 \\
\hline 17 & 1 & 5 & $5-6$ & 9.7 & 0.0 & 81.1 \\
\hline 17 & 2 & 1 & $20-21$ & 10.9 & 0.2 & 89.4 \\
\hline 17 & 2 & 2 & $44-45$ & 11.1 & 0.0 & 92.5 \\
\hline
\end{tabular}


TABLE 1 - Continued

\begin{tabular}{|c|c|c|c|c|c|c|}
\hline Hole & Core & Section & $\begin{array}{r}\text { Sampled } \\
\text { at }(\mathrm{cm})\end{array}$ & $\begin{array}{c}\text { Total Carbon } \\
\text { Per Cent }\end{array}$ & $\begin{array}{l}\text { Organic Carbon } \\
\text { Per Cent }\end{array}$ & $\begin{array}{c}\text { Calcium Carbonate } \\
\text { Per Cent }\end{array}$ \\
\hline 17 & 2 & 3 & $5-6$ & 10.9 & 0.2 & 89.3 \\
\hline 17 & 2 & 4 & $5-6$ & 11.2 & 0.1 & 92.5 \\
\hline 17 & 2 & 5 & $5-6$ & 10.3 & 0.1 & 84.6 \\
\hline 17 & 2 & 6 & $5-6$ & 10.0 & 0.1 & 82.8 \\
\hline $17 \mathrm{~A}$ & 1 & 1 & $11-12$ & 9.9 & 0.0 & 82.5 \\
\hline $17 \mathrm{~A}$ & 1 & 2 & $5-6$ & 10.7 & 0.5 & 85.1 \\
\hline $17 \mathrm{~A}$ & 1 & 3 & $5-6$ & 8.3 & 0.0 & 69.1 \\
\hline $17 \mathrm{~A}$ & 1 & 4 & $49-50$ & 6.8 & 0.2 & 54.8 \\
\hline $17 \mathrm{~A}$ & 1 & 5 & $5-6$ & 7.7 & 0.1 & 63.1 \\
\hline $17 \mathrm{~A}$ & 1 & 6 & $10-11$ & 9.0 & 0.0 & 74.6 \\
\hline $17 \mathrm{~A}$ & 2 & 1 & $5-6$ & 10.5 & 0.2 & 85.9 \\
\hline $17 \mathrm{~A}$ & 2 & 2 & $5-6$ & 10.0 & 0.2 & 81.5 \\
\hline $17 \mathrm{~A}$ & 2 & 3 & $5-6$ & 10.4 & 0.2 & 84.6 \\
\hline $17 \mathrm{~A}$ & 2 & 4 & $5-6$ & 9.6 & 0.1 & 79.0 \\
\hline $17 \mathrm{~A}$ & 2 & 5 & $5-6$ & 10.4 & 0.1 & 85.9 \\
\hline $17 \mathrm{~A}$ & 2 & 6 & $5-6$ & 10.0 & 0.1 & 82.2 \\
\hline $17 \mathrm{~A}$ & 3 & 1 & $5-6$ & 10.7 & 0.0 & 89.4 \\
\hline $17 \mathrm{~A}$ & 3 & 6 & $148-150$ & 10.7 & 0.2 & 87.5 \\
\hline $17 \mathrm{~A}$ & 4 & 2 & $3-4$ & 10.6 & 0.1 & 87.6 \\
\hline $17 \mathrm{~A}$ & 4 & 5 & $0-2$ & 10.9 & 1.0 & 82.6 \\
\hline $17 \mathrm{~A}$ & 4 & 6 & $4-5$ & 10.6 & 0.2 & 86.5 \\
\hline 17B & 1 & 1 & $5-6$ & 10.6 & 0.4 & 84.5 \\
\hline 17B & 1 & 2 & $5-6$ & 10.2 & 0.2 & 83.5 \\
\hline 17B & 1 & 3 & $5-6$ & 10.1 & 0.1 & 83.1 \\
\hline 17B & 1 & 4 & $5-6$ & 10.3 & 0.2 & 84.5 \\
\hline 17B & 1 & 5 & $30-31$ & 10.4 & 0.3 & 83.9 \\
\hline 17B & 1 & 6 & $45-46$ & 10.4 & 0.1 & 85.5 \\
\hline 17B & 2 & 1 & $5-6$ & 10.6 & 0.1 & 87.7 \\
\hline 17B & 2 & 2 & $5-6$ & 10.5 & 0.2 & 85.5 \\
\hline 17B & 2 & 3 & $5-6$ & 10.2 & 0.0 & 85.3 \\
\hline 17B & 2 & 4 & $5-6$ & 10.7 & 0.0 & 89.4 \\
\hline 17B & 2 & 5 & $5-6$ & 10.2 & 0.0 & 85.2 \\
\hline $17 \mathrm{~B}$ & 2 & 6 & $5-6$ & 10.2 & 0.0 & 85.0 \\
\hline 17B & 3 & 1 & $5-6$ & 9.7 & 0.2 & 79.0 \\
\hline 17B & 3 & 2 & $5-6$ & 10.8 & 0.0 & 89.6 \\
\hline 17B & 3 & 3 & $5-6$ & 10.6 & 0.1 & 87.3 \\
\hline 17B & 3 & 4 & $5-6$ & 9.0 & 0.0 & 74.6 \\
\hline 17B & 3 & 5 & $5-6$ & 9.6 & 0.1 & 78.9 \\
\hline 17B & 3 & 6 & $30-31$ & 9.6 & 0.0 & 80.2 \\
\hline
\end{tabular}


TABLE 1 - Continued

\begin{tabular}{|c|c|c|c|c|c|c|}
\hline Hole & Core & Section & $\begin{array}{r}\text { Sampled } \\
\text { at }(\mathrm{cm})\end{array}$ & $\begin{array}{c}\text { Total Carbon } \\
\text { Per Cent }\end{array}$ & $\begin{array}{l}\text { Organic Carbon } \\
\text { Per Cent }\end{array}$ & $\begin{array}{c}\text { Calcium Carbonate } \\
\text { Per Cent }\end{array}$ \\
\hline 17B & 4 & 1 & $30-31$ & 9.5 & 0.0 & 79.1 \\
\hline $17 \mathrm{~B}$ & 4 & 2 & $5-6$ & 10.3 & 0.0 & 86.1 \\
\hline 17B & 4 & 3 & $5-6$ & 10.4 & 0.0 & 86.6 \\
\hline 17B & 4 & 4 & $5-6$ & 9.8 & 0.1 & 80.6 \\
\hline 17B & 4 & 5 & $5-6$ & 10.5 & 0.0 & 87.6 \\
\hline $17 \mathrm{~B}$ & 4 & 6 & $10-11$ & 10.6 & 0.0 & 88.6 \\
\hline 18 & 1 & 5 & $20-21$ & 10.2 & 0.2 & 83.1 \\
\hline 18 & 2 & 1 & $0-2$ & 10.0 & 0.2 & 81.5 \\
\hline 18 & 2 & 2 & $5-6$ & 9.9 & 0.1 & 81.9 \\
\hline 18 & 2 & 3 & $148-150$ & 9.3 & 0.1 & 76.9 \\
\hline 18 & 2 & 4 & $148-150$ & 9.5 & 0.2 & 77.1 \\
\hline 18 & 2 & 5 & $148-150$ & 9.3 & 0.0 & 77.0 \\
\hline 18 & 2 & 6 & $?$ & 10.5 & 0.0 & 87.2 \\
\hline 18 & 3 & 1 & $148-150$ & 10.4 & 0.0 & 86.9 \\
\hline 18 & 3 & 2 & $5-6$ & 10.3 & 0.0 & 85.5 \\
\hline 18 & 3 & 3 & $148-150$ & 9.9 & 0.0 & 81.8 \\
\hline 18 & 3 & 4 & $148-150$ & 9.9 & 0.2 & 80.5 \\
\hline 18 & 3 & 5 & $148-150$ & 10.5 & 0.1 & 86.7 \\
\hline 18 & 3 & 6 & $148-150$ & 9.6 & 0.1 & 79.4 \\
\hline 18 & 4 & 2 & $0-2$ & 10.4 & 0.1 & 86.0 \\
\hline 18 & 4 & 3 & $0-2$ & 9.9 & 0.2 & 80.6 \\
\hline 18 & 4 & 4 & $5-6$ & 10.3 & 0.1 & 85.1 \\
\hline 18 & 4 & 5 & $148-150$ & 10.0 & 0.2 & 81.6 \\
\hline 18 & 5 & 1 & $148-150$ & 11.0 & 0.2 & 90.0 \\
\hline 18 & 5 & 3 & $148-150$ & 10.5 & 0.2 & 85.6 \\
\hline 18 & 5 & 5 & $0-2$ & 9.3 & 0.0 & 77.8 \\
\hline 18 & 5 & 6 & $0-2$ & 10.0 & 0.1 & 82.5 \\
\hline 18 & 6 & 1 & $5-6$ & 10.1 & 0.1 & 83.1 \\
\hline 18 & 6 & 2 & $148-150$ & 9.2 & 0.1 & 75.5 \\
\hline 18 & 6 & 3 & $148-150$ & 9.6 & 0.2 & 78.6 \\
\hline 18 & 6 & 4 & $148-150$ & 10.2 & 0.1 & 84.5 \\
\hline 18 & 6 & 6 & $3-4$ & 10.2 & 0.1 & 84.2 \\
\hline 19 & 1 & 1 & $40-41$ & 0.0 & 0.0 & 0.0 \\
\hline 19 & 1 & 2 & $5-6$ & 0.0 & 0.0 & 0.0 \\
\hline 19 & 1 & 3 & $4-5$ & 0.0 & 0.0 & 0.0 \\
\hline 19 & 1 & 4 & $5-6$ & 0.0 & 0.0 & 0.0 \\
\hline 19 & 1 & 5 & $5-6$ & 0.0 & 0.0 & 0.0 \\
\hline 19 & 1 & 6 & $5-6$ & 0.0 & 0.0 & 0.0 \\
\hline
\end{tabular}


TABLE 1 - Continued

\begin{tabular}{|c|c|c|c|c|c|c|}
\hline Hole & Core & Section & $\begin{array}{l}\text { Sampled } \\
\text { at }(\mathrm{cm})\end{array}$ & $\begin{array}{c}\text { Total Carbon } \\
\text { Per Cent }\end{array}$ & $\begin{array}{l}\text { Organic Carbon } \\
\text { Per Cent }\end{array}$ & $\begin{array}{c}\text { Calcium Carbonate } \\
\text { Per Cent }\end{array}$ \\
\hline 19 & 2 & 1 & $18-19$ & 0.0 & 0.0 & 0.0 \\
\hline 19 & 2 & 2 & $5-6$ & 0.0 & 0.0 & 0.0 \\
\hline 19 & 3 & 1 & $20-21$ & 7.9 & 0.1 & 65.1 \\
\hline 19 & 3 & 2 & $5-6$ & 4.3 & 0.0 & 36.2 \\
\hline 19 & 3 & 3 & $5-6$ & 6.3 & 0.0 & 52.1 \\
\hline 19 & 3 & 4 & $5-6$ & 2.7 & 0.0 & 22.4 \\
\hline 19 & 3 & 5 & $45-46$ & 7.3 & 0.0 & 60.7 \\
\hline 19 & 3 & 6 & $5-6$ & 2.4 & 0.0 & 20.3 \\
\hline 19 & 4 & 1 & $148-150$ & 7.1 & 0.0 & 59.3 \\
\hline 19 & 4 & 2 & $20-21$ & 9.1 & 0.0 & 76.0 \\
\hline 19 & 4 & 3 & $148-150$ & 10.0 & 0.0 & 83.5 \\
\hline 19 & 4 & 4 & $148-150$ & 9.3 & 0.0 & 77.3 \\
\hline 19 & 4 & 5 & $148-150$ & 9.3 & 0.0 & 77.6 \\
\hline 19 & 4 & 6 & $148-150$ & 8.6 & 0.2 & 70.6 \\
\hline 19 & 5 & 1 & $148-150$ & 9.5 & 0.2 & 77.1 \\
\hline 19 & 5 & 2 & $5-6$ & 9.1 & 0.2 & 74.4 \\
\hline 19 & 5 & 3 & $50-51$ & 7.8 & 0.2 & 63.6 \\
\hline 19 & 5 & 4 & $21-22$ & 9.0 & 0.0 & 75.0 \\
\hline 19 & 5 & 5 & $8-9$ & 7.5 & 0.1 & 61.6 \\
\hline 19 & 5 & 6 & $5-6$ & 8.7 & 0.0 & 72.5 \\
\hline 19 & 6 & 1 & $148-150$ & 8.7 & 0.0 & 72.6 \\
\hline 19 & 6 & 2 & $148-150$ & 8.2 & 0.0 & 67.0 \\
\hline 19 & 6 & 3 & $148-150$ & 8.6 & 0.1 & 71.1 \\
\hline 19 & 6 & 4 & $148-150$ & 7.0 & 0.0 & 58.1 \\
\hline 19 & 6 & 5 & $148-150$ & 9.5 & 0.0 & 79.2 \\
\hline 19 & 6 & 6 & $148-150$ & 8.3 & 0.0 & 68.9 \\
\hline 19 & 7 & 1 & $5-6$ & 7.7 & 0.0 & 63.9 \\
\hline 19 & 7 & 2 & $5-6$ & 8.7 & 0.3 & 70.1 \\
\hline 19 & 7 & 3 & $5-6$ & 8.7 & 0.0 & 72.5 \\
\hline 19 & 7 & 4 & $3-4$ & 6.4 & 0.0 & 53.1 \\
\hline 19 & 7 & 5 & $5-6$ & 9.2 & 0.1 & 76.4 \\
\hline 19 & 7 & 6 & $5-6$ & 9.4 & 0.1 & 77.8 \\
\hline 19 & 8 & 1 & $148-150$ & 9.8 & 0.2 & 79.6 \\
\hline 19 & 8 & 2 & 5-6 & 9.8 & 0.4 & 78.6 \\
\hline 19 & 8 & 3 & $148-150$ & 9.4 & 0.7 & 72.8 \\
\hline 19 & 8 & 4 & $148-150$ & 9.7 & 0.0 & 81.0 \\
\hline 19 & 8 & 5 & $148-150$ & 8.1 & 0.0 & 66.7 \\
\hline 19 & 8 & 6 & $148-150$ & 10.3 & 0.5 & 81.6 \\
\hline 19 & 9 & 1 & $148-150$ & 9.9 & 0.0 & 87.8 \\
\hline
\end{tabular}


TABLE 1 - Continued

\begin{tabular}{|c|c|c|c|c|c|c|}
\hline Hole & Core & Section & $\begin{array}{l}\text { Sampled } \\
\text { at }(\mathrm{cm})\end{array}$ & $\begin{array}{l}\text { Total Carbon } \\
\text { Per Cent }\end{array}$ & $\begin{array}{l}\text { Organic Carbon } \\
\text { Per Cent }\end{array}$ & $\begin{array}{c}\text { Calcium Carbonate } \\
\text { Per Cent }\end{array}$ \\
\hline 19 & 9 & 2 & $148-150$ & 9.9 & 0.0 & 82.1 \\
\hline 19 & 9 & 3 & $148-150$ & 9.7 & 0.4 & 77.6 \\
\hline 19 & 9 & 4 & $148-150$ & 9.8 & 0.3 & 79.1 \\
\hline 19 & 9 & 5 & $148-150$ & 9.4 & 0.1 & 77.1 \\
\hline 19 & 9 & 6 & $11-12$ & 9.2 & 0.0 & 76.8 \\
\hline 19 & 10 & 1 & $148-150$ & 10.0 & 0.0 & 82.9 \\
\hline 19 & 10 & 2 & $148-150$ & 8.7 & 0.0 & 72.6 \\
\hline 19 & 10 & 3 & $148-150$ & 9.7 & 0.0 & 80.6 \\
\hline 19 & 10 & 5 & $148-150$ & 9.9 & 0.2 & 80.5 \\
\hline 19 & 10 & 6 & $148-150$ & 9.8 & 0.2 & 79.8 \\
\hline 19 & 11 & 1 & $148-150$ & 9.5 & 0.0 & 79.3 \\
\hline 19 & 11 & 2 & $148-150$ & 9.3 & 0.0 & 77.3 \\
\hline 19 & 11 & 3 & $148-150$ & 9.3 & 0.0 & 77.4 \\
\hline 19 & 11 & 4 & $148-150$ & 9.9 & 0.0 & 82.4 \\
\hline $20 \mathrm{~A}$ & 2 & 1 & $50-51$ & 7.4 & 0.0 & 61.8 \\
\hline $20 \mathrm{~A}$ & 2 & 2 & $5-6$ & 8.4 & 0.0 & 69.8 \\
\hline $20 \mathrm{~A}$ & 2 & 3 & $10-11$ & 8.7 & 0.1 & 71.4 \\
\hline $20 \mathrm{~A}$ & 2 & 4 & $148-150$ & 8.8 & 0.2 & 71.9 \\
\hline $20 \mathrm{~A}$ & 2 & 5 & $148-150$ & 9.1 & 0.4 & 72.9 \\
\hline $20 \mathrm{~A}$ & 2 & 6 & $148-150$ & 10.5 & 0.0 & 87.5 \\
\hline $20 \mathrm{~B}$ & 1 & 1 & $5-6$ & 2.6 & 0.0 & 21.5 \\
\hline $20 \mathrm{~B}$ & 1 & 2 & $10-11$ & 2.6 & 0.0 & 21.6 \\
\hline $20 \mathrm{~B}$ & 1 & 3 & $10-11$ & 4.4 & 0.0 & 36.8 \\
\hline $20 \mathrm{~B}$ & 1 & 4 & $5-6$ & 4.9 & 0.0 & 40.7 \\
\hline 20B & 1 & 5 & $5-6$ & 4.1 & 0.2 & 32.2 \\
\hline $20 \mathrm{~B}$ & 1 & 6 & $25-26$ & 9.3 & 0.1 & 76.1 \\
\hline $20 \mathrm{~B}$ & 1 & 6 & $100-110$ & 7.3 & 0.2 & 59.6 \\
\hline $20 \mathrm{C}$ & 1 & 2 & $49-50$ & 0.0 & 0.0 & 0.0 \\
\hline $20 \mathrm{C}$ & 1 & 3 & $3-4$ & 4.5 & 0.0 & 37.2 \\
\hline $20 \mathrm{C}$ & 1 & 4 & $5-6$ & 5.2 & 0.1 & 42.5 \\
\hline $20 \mathrm{C}$ & 1 & 5 & $43-44$ & 3.7 & 0.0 & 31.2 \\
\hline $20 \mathrm{C}$ & 1 & 6 & $5-6$ & 2.8 & 0.0 & 23.4 \\
\hline $20 \mathrm{C}$ & 2 & 1 & $148-150$ & 10.0 & 2.1 & 66.1 \\
\hline $20 \mathrm{C}$ & 2 & 2 & $148-150$ & 10.2 & 0.0 & 84.8 \\
\hline $20 \mathrm{C}$ & 2 & 3 & $29-30$ & 9.5 & 2.9 & 54.8 \\
\hline $20 \mathrm{C}$ & 2 & 4 & $47-48$ & 10.3 & 3.2 & 59.7 \\
\hline $20 \mathrm{C}$ & 2 & 5 & $148-150$ & 9.8 & 1.3 & 71.0 \\
\hline $20 \mathrm{C}$ & 2 & 6 & $148-150$ & 10.2 & 1.5 & 72.1 \\
\hline $20 \mathrm{C}$ & 3 & 1 & $5-6$ & 5.3 & 0.1 & 44.0 \\
\hline
\end{tabular}


TABLE 1 - Continued

\begin{tabular}{|c|c|c|c|c|c|c|}
\hline Hole & Core & Section & $\begin{array}{l}\text { Sampled } \\
\text { at }(\mathrm{cm})\end{array}$ & $\begin{array}{l}\text { Total Carbon } \\
\text { Per Cent }\end{array}$ & $\begin{array}{l}\text { Organic Carbon } \\
\text { Per Cent }\end{array}$ & $\begin{array}{c}\text { Calcium Carbonate } \\
\text { Per Cent }\end{array}$ \\
\hline $20 \mathrm{C}$ & 3 & 2 & $5-6$ & 3.7 & 0.3 & 28.4 \\
\hline $20 \mathrm{C}$ & 3 & 3 & $10-11$ & 2.3 & 0.3 & 16.0 \\
\hline $20 \mathrm{C}$ & 3 & 4 & $12-13$ & 3.7 & 0.0 & 31.0 \\
\hline $20 \mathrm{C}$ & 3 & 5 & $120-121$ & 6.3 & 0.0 & 52.4 \\
\hline $20 \mathrm{C}$ & 3 & 6 & $5-6$ & 4.3 & 0.0 & 36.0 \\
\hline $20 \mathrm{C}$ & 4 & 1 & $21-22$ & 4.0 & 0.0 & 33.1 \\
\hline $20 \mathrm{C}$ & 4 & 2 & $5-6$ & 3.7 & 0.0 & 31.1 \\
\hline $20 \mathrm{C}$ & 4 & 3 & $48-49$ & 4.2 & 0.0 & 35.0 \\
\hline $20 \mathrm{C}$ & 4 & 4 & $148-150$ & 4.0 & 0.0 & 33.5 \\
\hline $20 \mathrm{C}$ & 5 & 1 & $148-150$ & 9.1 & 0.0 & 75.5 \\
\hline $20 \mathrm{C}$ & 5 & 3 & $50-51$ & 8.6 & 0.3 & 69.3 \\
\hline $20 \mathrm{C}$ & 5 & 4 & $46-47$ & 7.8 & 0.0 & 64.6 \\
\hline $20 \mathrm{C}$ & 5 & 5 & $5-6$ & 10.6 & 0.0 & 88.5 \\
\hline $20 \mathrm{C}$ & 5 & 6 & $5-6$ & 10.6 & 0.0 & 87.9 \\
\hline $20 \mathrm{C}$ & 6 & 1 & $148-150$ & 7.3 & 0.0 & 61.0 \\
\hline $20 \mathrm{C}$ & 6 & 2 & $3-4$ & 9.5 & 0.0 & 79.0 \\
\hline $20 \mathrm{C}$ & 6 & 3 & $46-47$ & 10.0 & 0.1 & 82.4 \\
\hline $20 \mathrm{C}$ & 6 & 4 & $36-40$ & 9.1 & 0.0 & 75.8 \\
\hline $20 \mathrm{C}$ & 6 & 5 & $5-6$ & 7.3 & 0.1 & 59.4 \\
\hline 21 & 1 & 1 & $148-150$ & 8.8 & 0.0 & 73.5 \\
\hline 21 & 1 & 2 & $148-150$ & 10.4 & 0.0 & 86.7 \\
\hline 21 & 1 & 3 & $148-150$ & 11.4 & 0.0 & 94.7 \\
\hline 21 & 1 & 4 & $27-28$ & 9.9 & 0.0 & 82.6 \\
\hline 21 & 1 & 5 & $148-150$ & 10.5 & 0.2 & 85.7 \\
\hline 21 & 1 & 6 & $148-150$ & 7.5 & 0.0 & 62.6 \\
\hline 21 & 2 & 1 & $148-150$ & 10.1 & 0.0 & 84.5 \\
\hline 21 & 2 & 2 & $0-3$ & 9.6 & 0.0 & 79.9 \\
\hline 21 & 2 & 3 & $148-150$ & 8.6 & 0.0 & 71.3 \\
\hline 21 & 2 & 4 & $5-6$ & 10.4 & 0.0 & 87.0 \\
\hline 21 & 2 & 6 & $5-6$ & 10.4 & 0.0 & 86.2 \\
\hline 21 & 3 & 1 & $148-150$ & 8.9 & 0.1 & 73.0 \\
\hline 21 & 3 & 2 & $148-150$ & 8.7 & 0.0 & 72.7 \\
\hline 21 & 3 & 3 & $148-150$ & 9.3 & 0.1 & 76.6 \\
\hline 21 & 3 & 4 & $148-150$ & 9.3 & 0.1 & 76.6 \\
\hline 21 & 3 & 6 & $148-150$ & 8.4 & 0.0 & 70.1 \\
\hline 21 & 4 & 1 & $148-150$ & 8.2 & 0.1 & 67.8 \\
\hline 21 & 4 & 2 & $148-150$ & 8.9 & 0.1 & 73.1 \\
\hline 21 & 4 & 3 & $148-150$ & 9.5 & 0.2 & 77.1 \\
\hline 21 & 4 & 5 & $148-150$ & 8.5 & 0.0 & 70.8 \\
\hline
\end{tabular}


TABLE 1 - Continued

\begin{tabular}{|c|c|c|c|c|c|c|}
\hline Hole & Core & Section & $\begin{array}{l}\text { Sampled } \\
\text { at }(\mathrm{cm})\end{array}$ & $\begin{array}{c}\text { Total Carbon } \\
\text { Per Cent }\end{array}$ & $\begin{array}{l}\text { Organic Carbon } \\
\text { Per Cent }\end{array}$ & $\begin{array}{c}\text { Calcium Carbonate } \\
\text { Per Cent }\end{array}$ \\
\hline 21 & 4 & 6 & $148-150$ & 9.7 & 0.1 & 79.9 \\
\hline 21 & 5 & 1 & $5-6$ & 8.8 & 0.0 & 73.2 \\
\hline 21 & 5 & 2 & $148-150$ & 9.7 & 0.3 & 78.3 \\
\hline 21 & 5 & 4 & $148-150$ & 6.4 & 0.2 & 52.4 \\
\hline 21 & 5 & 6 & $148-150$ & 7.4 & 0.0 & 62.0 \\
\hline 21 & 6 & 3 & $15-16$ & 9.1 & 0.0 & 75.5 \\
\hline 21 & 6 & 4 & $5-6$ & 8.6 & 0.0 & 71.6 \\
\hline 21 & 6 & 5 & $9-10$ & 7.9 & 0.0 & 65.5 \\
\hline 21 & 6 & 6 & $8-9$ & 9.2 & 0.1 & 75.8 \\
\hline 21 & 7 & 1 & $148-150$ & 9.1 & 0.0 & 75.7 \\
\hline 21 & 7 & 2 & $148-150$ & 9.6 & 0.1 & 79.1 \\
\hline 21 & 7 & 3 & $148-150$ & 9.4 & 0.1 & 77.2 \\
\hline 21 & 7 & 4 & $148-150$ & 9.7 & 0.1 & 79.8 \\
\hline 21 & 7 & 5 & $148-150$ & 9.6 & 0.1 & 79.1 \\
\hline 21 & 7 & 6 & $148-150$ & 9.5 & 0.0 & 79.0 \\
\hline 21 & 8 & 1 & $0-2$ & 9.6 & 0.0 & 79.9 \\
\hline 21 & 8 & 2 & $148-150$ & 9.6 & 0.0 & 79.8 \\
\hline 21 & 8 & 3 & $148-150$ & 10.0 & 0.0 & 83.1 \\
\hline 21 & 8 & 4 & $148-150$ & 9.6 & 0.0 & 80.0 \\
\hline 21 & 8 & 5 & $148-150$ & 9.9 & 1.3 & 71.7 \\
\hline $21 \mathrm{~A}$ & 1 & 1 & $148-150$ & 9.8 & 0.7 & 75.4 \\
\hline $21 \mathrm{~A}$ & 1 & 2 & $148-150$ & 10.6 & 0.6 & 83.4 \\
\hline $21 \mathrm{~A}$ & 1 & 3 & $148-150$ & 7.0 & 0.1 & 57.5 \\
\hline $21 \mathrm{~A}$ & 1 & 4 & $148-150$ & 10.4 & 0.5 & 81.6 \\
\hline $21 \mathrm{~A}$ & 1 & 5 & $148-150$ & 9.2 & 0.0 & 76.4 \\
\hline $21 \mathrm{~A}$ & 1 & 6 & $148-150$ & 10.0 & 0.0 & 83.0 \\
\hline $21 \mathrm{~A}$ & 2 & 1 & $148-150$ & 10.5 & 0.0 & 87.3 \\
\hline $21 \mathrm{~A}$ & 2 & 2 & $148-150$ & 9.5 & 0.2 & 77.7 \\
\hline $21 \mathrm{~A}$ & 2 & 3 & $148-150$ & 11.0 & 0.3 & 89.1 \\
\hline $21 \mathrm{~A}$ & 2 & 5 & $148-150$ & 11.0 & 0.6 & 86.6 \\
\hline $21 \mathrm{~A}$ & 2 & 6 & $148-150$ & 10.8 & 0.1 & 89.3 \\
\hline $21 \mathrm{~A}$ & 3 & 1 & $148-150$ & 10.1 & 0.2 & 82.2 \\
\hline $21 \mathrm{~A}$ & 3 & 2 & $148-150$ & 10.1 & 0.0 & 83.8 \\
\hline $21 \mathrm{~A}$ & 3 & 3 & $148-150$ & 10.3 & 0.0 & 85.6 \\
\hline $21 \mathrm{~A}$ & 3 & 4 & $148-150$ & 10.1 & 0.0 & 84.0 \\
\hline $21 \mathrm{~A}$ & 3 & 6 & $60-61$ & 9.9 & 0.0 & 82.1 \\
\hline 22 & 1 & 1 & $148-150$ & 9.7 & 0.3 & 78.3 \\
\hline 22 & 1 & 2 & $148-150$ & 10.2 & 0.0 & 84.9 \\
\hline 22 & 1 & 3 & $148-150$ & 10.4 & 0.0 & 86.6 \\
\hline
\end{tabular}


TABLE 1 - Continued

\begin{tabular}{l|c|c|c|c|c|c}
\hline Hole & Core & Section & $\begin{array}{c}\text { Sampled } \\
\text { at (cm) }\end{array}$ & $\begin{array}{c}\text { Total Carbon } \\
\text { Per Cent }\end{array}$ & $\begin{array}{c}\text { Organic Carbon } \\
\text { Per Cent }\end{array}$ & $\begin{array}{c}\text { Calcium Carbonate } \\
\text { Per Cent }\end{array}$ \\
\hline 22 & 1 & 4 & $148-150$ & 10.0 & 0.0 & 83.5 \\
22 & 1 & 6 & $26-27$ & 10.5 & 0.0 & 87.5 \\
22 & 2 & 1 & $10-11$ & 9.8 & 0.0 & 81.1 \\
22 & 2 & 2 & $4-5$ & 10.0 & 0.1 & 82.5 \\
22 & 2 & 3 & $13-14$ & 9.6 & 0.1 & 79.9 \\
22 & 2 & 6 & $5-6$ & 10.4 & 0.3 & 84.1 \\
22 & 3 & 1 & $148-150$ & 9.2 & 0.2 & 74.3 \\
22 & 3 & 3 & $148-150$ & 9.6 & 3.9 & 47.5 \\
22 & 3 & 4 & $148-150$ & 9.0 & 1.1 & 66.6 \\
22 & 3 & 5 & $148-150$ & 10.8 & 0.3 & 87.4 \\
22 & 3 & 6 & $50-51$ & 7.5 & 0.1 & 61.8 \\
22 & 4 & 2 & $74-75$ & 10.9 & 0.2 & 82.4 \\
22 & 4 & 3 & $148-150$ & 8.3 & 0.1 & 81.0 \\
22 & 4 & 4 & $5-6$ & 9.3 & 0.1 & 88.6 \\
22 & 4 & 5 & $5-6$ & 10.9 & 0.3 & 89.1 \\
22 & 4 & 6 & $10-11$ & 10.7 & 0.0 & \\
\hline
\end{tabular}

TABLE 2A

Average Carbonate Values from Leg 3 Holes

\begin{tabular}{|c|c|c|c|c|c|}
\hline Hole & $\begin{array}{l}\text { Number of } \\
\text { Samples } \\
\text { Averaged }\end{array}$ & $\begin{array}{l}\text { Average } \\
\text { Carbonate } \\
\text { Per Cent }\end{array}$ & $\begin{array}{l}\text { Position } \\
\text { of Hole }\end{array}$ & $\begin{array}{l}\text { Ocean Depth } \\
\text { at Present } \\
\text { (meters) }\end{array}$ & $\begin{array}{l}\text { Age of Most } \\
\text { of Sediment }\end{array}$ \\
\hline 14 & 21 & 71.38 & $\begin{array}{l}\text { West of } \\
\text { ridge }\end{array}$ & 4222 & Oligocene-Miocene \\
\hline 15 & 47 & 71.46 & $\begin{array}{l}\text { West of } \\
\text { ridge }\end{array}$ & 3830 & Miocene-Pliocene \\
\hline 16 & 58 & 88.87 & $\begin{array}{l}\text { Crest of } \\
\text { ridge }\end{array}$ & 3433 & Upper Miocene-Pliocene \\
\hline 17 & 52 & 83.51 & $\begin{array}{l}\text { East of } \\
\text { ridge }\end{array}$ & 4143 & Upper Oligocene-Miocene \\
\hline 18 & 26 & 82.33 & $\begin{array}{l}\text { East of } \\
\text { ridge }\end{array}$ & 3920 & Miocene \\
\hline $19^{\mathrm{a}}$ & 45 & 74.53 & $\begin{array}{l}\text { Far west } \\
\text { of ridge }\end{array}$ & 4544 & Eocene-Oligocene \\
\hline $20^{\mathrm{a}}$ & 6 & 72.55 & $\begin{array}{l}\text { Far west } \\
\text { of ridge }\end{array}$ & 4319 & Upper Cretaceous-Oligocene \\
\hline
\end{tabular}


TABLE 2A - Continued

\begin{tabular}{|c|c|c|c|c|c|}
\hline Hole & $\begin{array}{c}\text { Number of } \\
\text { Samples } \\
\text { Averaged }\end{array}$ & $\begin{array}{l}\text { Average } \\
\text { Carbonate } \\
\text { Per Cent }\end{array}$ & $\begin{array}{l}\text { Position } \\
\text { of Hole }\end{array}$ & $\begin{array}{l}\text { Ocean Depth } \\
\text { at Present } \\
\text { (meters) }\end{array}$ & $\begin{array}{l}\text { Age of Most } \\
\text { of Sediment }\end{array}$ \\
\hline $20^{\mathrm{a}}$ & 10 & 74.34 & $\begin{array}{l}\text { Far west } \\
\text { of ridge }\end{array}$ & 4319 & Upper Cretaceous-Oligocene \\
\hline 21 & 56 & 77.78 & $\begin{array}{l}\text { Rio Grande } \\
\text { Rise }\end{array}$ & 2064 & Upper Cretaceous-Pliocene \\
\hline 22 & 19 & 78.68 & $\begin{array}{l}\text { Rio Grande } \\
\text { Rise }\end{array}$ & 2106 & Eocene-Miocene \\
\hline
\end{tabular}

TABLE 2B

Leg 2 Results for Comparison

\begin{tabular}{|c|c|c|c|c|c|}
\hline $10^{\mathrm{a}}$ & 44 & 69.00 & $\begin{array}{l}\text { Far west } \\
\text { of ridge }\end{array}$ & 4712 & Upper Cretaceous-Pliocene \\
\hline 11 & 8 & 82.00 & $\begin{array}{l}\text { Crest of } \\
\text { ridge }\end{array}$ & 3571 & Miocene-Pliocene \\
\hline
\end{tabular}

${ }^{a}$ Sections of brown clay with low to nil carbonate values have been excluded from the figures averaged for carbonate per cent.

The results, therefore, indicate that the lower carbonate values on the west side of the ridge in Tertiary times were probably caused by partial solution of calcite by the Antarctic bottom water. The complete carbonate results in Table 1 show that Cores 1 and 2 from Hole 19 were in red clay with no carbonate, but from Core 3 downward the carbonate percentage increases. This indicates that at present times the area of Hole 19 is below the carbonate compensation depth; but, previously, it probably underwent partial solution of carbonate content and where the highest values occur no solution. The water must have been progressively deepening over this site; in fact, because of sea floor spreading the site itself probably moved west down the flank of the Mid-Atlantic Ridge into deeper water. The results of Hole 14 indicate that there is also partial solution of calcite in Core 1 (Lower Miocene), so here again is evidence of progressive increase in water depth over a particular site.

Hole 13 -on the Sierra Leone Rise penetrated brown clay and radiolarian ooze with a low carbonate content of 0.5 to 5 per cent, and also marl and chalk oozes with carbonate values up to 63 per cent.

It can be seen from Table 3 that the content of organic carbon in sediments from Leg 3 was extremely low, in most places averaging about 0.1 per cent. In a few places anomalously high readings were recorded as follows: Hole 20C, Core 2 averages 1.8 per cent organic

\section{ORGANIC CARBON}

TABLE 3

Organic Carbon Results

\begin{tabular}{lcc}
\hline Hole & $\begin{array}{r}\text { Average Per Cent } \\
\text { Organic Carbon }\end{array}$ & $\begin{array}{c}\text { Sections with } \\
\text { Anomalous } \\
\text { Values Excluded }\end{array}$ \\
\hline 13 & 0.3 & - \\
14 & 0.1 & - \\
15 & $<0.1$ & - \\
16 & $<0.1$ & - \\
17 & 0.1 & $17 \mathrm{~A}-4-5$ \\
18 & 0.1 & - \\
19 & $<0.1$ & - \\
$20 \mathrm{~A}, \mathrm{~B}$ & 0.1 & - \\
$20 \mathrm{C}$ & $<0.1$ & $20 \mathrm{C}-2-1 \& 6$ \\
21 & $<0.1$ & $21-8-5$ \\
$21 \mathrm{~A}$ & 0.2 & - \\
22 & 0.1 & $22-3-3 \& 4$ \\
\hline
\end{tabular}

carbon with one value of over 3 per cent; Hole 22, Core 3 , Sections 3 and 4 have organic carbon values of 3.9 and 1.1 per cent, respectively. Judging from the original core descriptions, there is no apparent difference in sediment type at these horizons to explain these high values. 